\title{
Application of the WNN-Based SCG Optimization Algorithm for Predicting Soft Soil Foundation Engineering Settlement
}

\author{
Guihua Li (i), Chenyu Han (D), Hong Mei, and Shuai Chen \\ School of Earth Sciences and Engineering, Hohai University, Nanjing 210098, China \\ Correspondence should be addressed to Chenyu Han; 191309020011@hhu.edu.cn
}

Received 10 August 2021; Revised 8 September 2021; Accepted 15 September 2021; Published 14 October 2021

Academic Editor: Mian Ahmad Jan

Copyright (C) 2021 Guihua Li et al. This is an open access article distributed under the Creative Commons Attribution License, which permits unrestricted use, distribution, and reproduction in any medium, provided the original work is properly cited.

\begin{abstract}
Settlement prediction in soft soil foundation engineering is a newer technique. Predicting soft soil settling has long been one of the most challenging techniques due to difficulties in soft soil engineering. To overcome these challenges, the wavelet neural network (WNN) is mostly used. So, after assessing its estimate performance, two elements, early parameter selection and system training techniques, are chosen to optimize the traditional WNN difficulties of readily convergence to the local infinitesimal point, low speed, and poor approximation performance. The number of hidden layer nodes is determined using a self-adaptive adjustment technique. The wavelet neural network (WNN) is coupled with the scaled conjugate gradient (SCG) to increase the feasibility and accuracy of the soft fundamental engineering settlement prediction model, and a better wavelet network for the soft ground engineering settlement prediction is suggested in this paper. Furthermore, we have proposed the technique of locating the early parameters based on autocorrelation. The settlement of three types of traditional soft foundation engineering, including metro tunnels, highways, and high-rise building foundations, has been predicted using our proposed model. The findings revealed that the model is superior to the backpropagation neural network and the standard WNN for solving problems of approximation performance. As a result, the model is acceptable for soft foundation engineering settlement prediction and has substantial project referential value.
\end{abstract}

\section{Introduction}

Prediction of soft soil settlement has always been one of the technical problems in soft soil engineering. As there are many factors affecting the settlement of soft foundations, how to predict the settlement of soft foundations correctly becomes a common problem for researchers in design and construction. To improve the accuracy, calculation and prediction based on measured data are a general method in engineering at present [1]. Commonly used deformation prediction methods based on measured data include statistical analysis, time series analysis, grey system theory, Kalman filter, and neural networks, but these have their limitations [2,3]. Optimizing the model and improving the prediction accuracy are an important content of the deformation prediction model. According to the actual application research, a single theory or model is difficult to accurately predict the magnitude of deformation. The close combination and comprehensive comparison of multiple theoretical models is an effective way to study the prediction of deformation [3, 4]. By integrating the advantages of wavelet analysis with artificial neural networks, the wavelet neural network (WNN) has been swiftly established and has played a key role in deformation monitoring. On the other hand, as the economy has grown, communities have seen an increase in the number of highrise structures. The settling of the building foundation must be checked and anticipated to assure its safety during the construction and operation phases. However, due to the difficulty of physical circumstances and the ambiguity of the causes driving settlement, using traditional certainty concepts to forecast settlement is extremely challenging. Although the inclusion of WNN simplifies the problem, it has several drawbacks in its current form.

The wavelet neural network (WNN) is a new exploration under this guiding ideology, which combines wavelet analysis and neural network successfully and thus provides a 
scientific theoretical basis and analysis tool for modern forecasting $[5,6]$. There are many forms of WNN. Compact wavelet neural networks that use wavelet functions to replace the hidden layer functions of conventional neural networks seem common, but they display convergence to local minima, low speed, and poor approximation performance frequently, and some other shortcomings as well [6-9]. Accordingly, the present study proposes an optimized WNN built on the scaled conjugate gradient algorithm (SCG). Within the study, the settlement data of metro tunnels, highway soft foundations and roads, and high-rise buildings are taken as soft foundation engineering examples. Three neural networks, BP neural network, the traditional WNN based on backpropagation (BP algorithm), and WNN based on SCG algorithm, are compared and analyzed comprehensively. This article designed an optimization of WNN built on the SCG method to predict soft soil foundation engineering settlement under the complex geology settings to overcome issues mentioned above. The results show that the optimization model achieves a better performance and is more suitable than the other two networks for soft soil foundation engineering settlement prediction.

The following are the offerings of this research work:

(1) We investigate the soft soil foundation engineering settlement prediction and wavelet neural network. We explain the methodology that we have adopted during our proposed work and perform experiment for our three techniques, i.e., BP Neural Network, WNN-based BP Algorithm, and Improved Wavelet Neural Network.

(2) We compare the prediction accuracy obtained during the three techniques mentioned above.

(3) Our proposed work improves the stability and convergence accuracy of WNN. Hence, the initial parameter setting method can be linked with wavelet type, wavelet time-frequency parameters, and learning samples.

(4) From our proposed scheme, we concluded that SCG algorithm combined with the autocorrelation correction can determine the number of hidden layer nodes. Thus, an improved WNN can be derived successfully.

The rest of the research work consists of the following: Section 2 explains the related work, Section 3 illustrates material and methodology used during our proposed work, Section 4 deliberates our experimental work that we have performed during our work, and finally, the paper is concluded in Section 5.

\section{Related Work}

Soft soil is found all around the world. Its unique characteristics, i.e., high void ratio, high water content, high compressibility, low shear strength, low permeability, and unique structural characteristics, necessitate particular consideration in the study, construction, and maintenance of geotechnical structures built on them. Large-scale construction of high-speed transit systems, high-rise structures, and subterranean works for numerous urban centers built on such soils is a huge problem. Many researchers have attempted to overcome the issues that arise in the foundation of soft soils. The authors in [10] used a threedimensional (3D) analytic approach to investigate the influence of pavement smoothness on the dynamic behavior of subsurface movement loads. The impact of unconventional and low-carbon additions on the long-term treatment of soils for building and paving materials is investigated. Data from three centrifuge tests during traffic loads are used to study the cyclic behavior of mud with a ground comprised of overcompacted soil at the top.

The researchers of [6] used a 3D dynamic finite-element analysis to estimate the permanent settling of a segment of the cross-river tunnel, allowing for the influence of primary stress rotation and verifications for test data. The disrupted state concept (DSC) model for normally consolidated clays was described in [11], which included the impacts of cracking, particle breaking, heating, softness, and hardening. The overconsolidation ratio on strength, militancy, and distortion is used to develop a novel disruption function. Consolidation theory, numerical computation, and curve fitting are three types of approaches for forecasting ground settlement. There have been several recent works on consolidation theory [12-14].

In a novel approach, the authors of [15] described the settlement of an inspired embankment on a soft basis based on a classic hyperbolic approach and used the system deformations features reflected in preloaded embankments to forecast the settlement during the later stage. In [16], the authors proposed a staged observational technique for predicting embankment settlement on soft ground with staged construction; they discovered that immediate settlement adds to the shift distance of the parallel lines during staged construction. The researchers in [17] used a genetic algorithm to optimize a BP neural network to forecast the summer electrical short-term load. Because of its high convergence rate and low memory use, the scaled conjugate gradient (SCG) method is used in NFC training. As a result, the authors of [18] trained a type-1 fuzzy system using a customized form of SCG. According to them, the improved SCG accelerates convergence in the steepest descent approach of fuzzy system training. As a consequence, the SCG appears to be a good candidate for NFC training for largescale issues. Training NFC with SCG for large-scale issues, on the other hand, might take days or weeks on any personal computer. Training NFC with SCG for large-scale issues, on the other hand, might take days or weeks on any personal computer. An alternate method for reducing training time is to calculate the Hessian matrix using first-order gradients, as in conjugate gradient (CG) algorithms [19]. A transformation wavelet is a useful tool for data processing and timefrequency representation development. The wavelet theories are described thoroughly in $[20,21]$. In the context of neural networks, the application of wavelet transform is not new. Previous research $[22,23]$ proposed a theoretical framework for neural feed-forward networks based on wavelets. The study of [24] has investigated the capacity to employ 
wavelet-based cross-pollination for an unknown real-time function. Because wavelets have a high compression capacity and have fewer coefficients, the results were achieved in this circumstance.

In [25], the authors offer a statistical model identification framework for using wavelet networks, which is studied over a wide range of topics, including architecture, initialization, variable selection, and model selection. Because of their capacity to extract varied information, wavelet-based techniques have been employed in numerous computer vision applications using Convolutional Neural Networks (CNN). Wavelet CNN texture classification [26], multiscale face superresolution [27], picture superresolution [28], and edge feature boosting [29] are only a few examples. In [30], a multilevel wavelet $\mathrm{CNN}$ model for picture restoration was presented. The researchers in [31] suggested a new layer that conducts wavelet-based convolution filtering and activation before returning to pixel space.

Similarly, [32] created a hybrid wavelet deep learning network based on the wavelets scattering transform [33]. This presented a basic classification that was subsequently enhanced [34]. In [35], the authors also suggest a wavelet for the segmentation of the brain tumors, which is strengthened by an evolutionary network design, and for this application, DWT is coupled with the neural network classification [36]. To lower the spatial resolution and expand the receptive field to dense pixel-specific prediction, encoder-decoder $\mathrm{CNN}$ architecture with encoder DWT and inverse decoder DWT was presented in [37]. In addition, a neural wavelet network for speech and noise separation was proposed in [38]. The researchers in [39] built WNN optimization based on the SCG algorithm to forecast the settling of the foundation of the structure under complex geological circumstances. The findings indicated that WNN optimization was optimal and that this had a positive effect compared to the BP neural network and the BP WNN. Inspired from the work of above scholars, this research work combines wavelet neural network (WNN) with optimized scaled conjugate gradient algorithm to successfully predict the soft soil foundation engineering settlement by performing numerous experiments.

\section{Materials and Methodology}

\subsection{Materials Used during Our Research Work}

3.1.1. Soft Soil Foundations. Soft soil foundation consists of soft soil with finer particles and organic soil with wider gaps, because its texture comprises mucky soil with silt and other highly compressible soils with clay components and silt components [40] in fine soil particles. The soft foundations of soil are mostly based on the changes in soil foundations produced by geographical circumstances, geological structures, and the soil conditions' features and qualities.

(1) Characteristics of Soft Soil Foundation. The major characteristics of soft soil include low water permeability and high water content, according to the basic attributes of soil. However, the water permeability is poor, and the shear strength is exceedingly low [41]. The shear foot brake and compression system with high compressibility or mucky soil generally exhibit considerable settlement after the external load is transmitted to the foundation section. As a result, constructions erected on soft soil foundations, such as buildings, roads, and bridges, have a significant inclination or settlement. It is straightforward to cause the injury and crack of the building, and therefore, the increase of the macrospore, which can cause the collapse of the building once it is serious. Therefore, the municipal construction units should actively analyze and study the soft soil foundation treatment and scientifically discover and take a look at the shear resistance and cargo resistance level of the soft soil foundation [42].

(2) The Impact of Soft Soil Foundation. The impact of soft soil foundation can be seen in Figure 1 and its major components are as follows:

(1) Poor bearing capacity: due to the high water content and tiny seepage of the soil conditions in soft soil foundations, the bearing strength of the foundation is fairly low, making settlement foundations extremely simple to produce, posing a severe danger to residents' travel quality [43].

(2) Large settlement: the significant settlement is one of the features of a soft soil foundation. The settling features of soft soil foundations will create additional difficulties for engineering building projects and pose a severe danger to the progress and quality of engineering projects.

(3) Strong compressibility: the soft soil ductility is strong, due to the macrospore structure depending on water content and soil quality [12]. During construction, the macrospore's soft soil layer is endangered, which prevents appropriate control measures from being taken and further impacts the efficiency and progress of the building and the stability of the base. The consequence is easy to dislocate the path and the subgrade of the city's engineering structure collapses.

3.1.2. Wavelet Neural Network. Wavelet Networks are a novel network type that brings together traditional Sigmoid Networks (NNs) with Wavelet Analysis (WA) [43]. The stretch factor, denoted by $a j$, and the panning element, denoted by $b j$, are two new parameters introduced by this technique. These new parameters replace the respective weights and thresholds of the neural network by using the wave element to replace neurons and establish a connection between the transforming and the neural networks through an approximation of wavelet decomposition [25]. The wavelet neural network contains three layers according to Figure 2: input layer, hidden layer, and layer output. During the forward propagation learning phase, the data from the input layer is processed and sent to the hidden layer. The data is subsequently processed in the output layer by a hidden layer. After that, at the backpropagation stage, the 


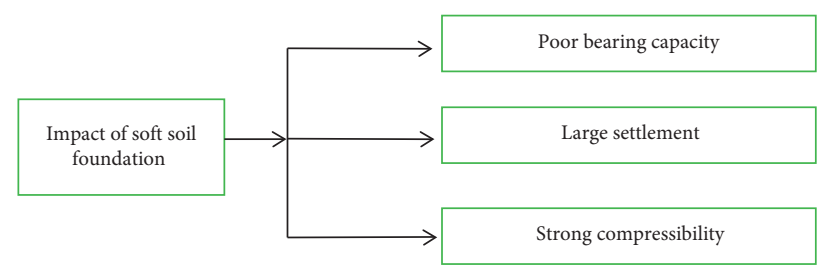

FIgUre 1: Impact of soft soil foundation.

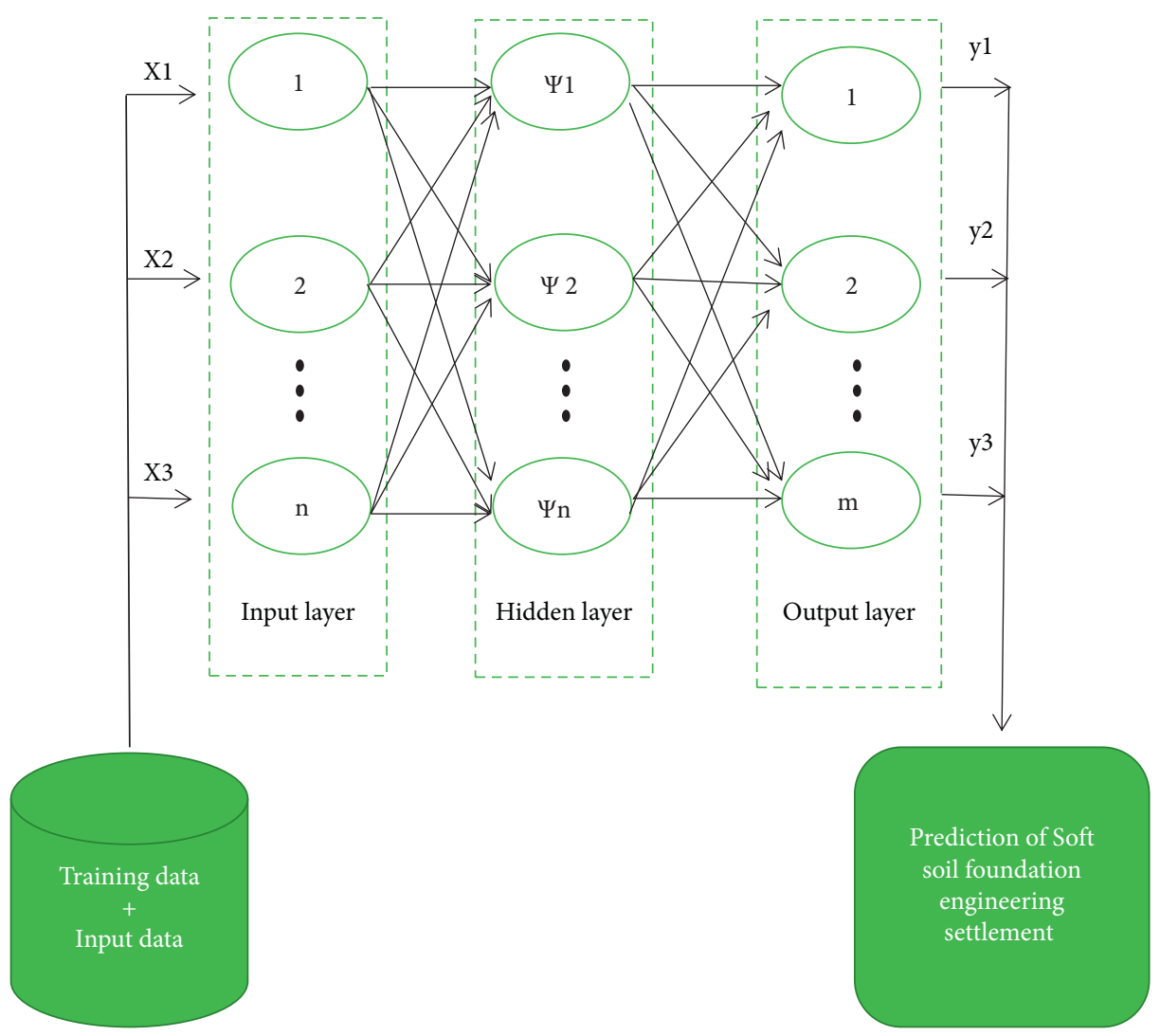

FIGURE 2: Wavelet neural network.

output layer determines the output value of each unit by computing the difference between the output value and the intended output values. Finally, the weight to modify each input layer and the hidden layer is the product of each receiving unit error value and transmission unit activation value $[44,45]$.

\subsection{Methodology}

3.2.1. Convergence Analysis. After considering the WNN's limitations, the main issue is poor convergence. When the wavelet-based neural network uses the BP neural network's initialization and training technique, there will be poor convergence difficulties and recommended improvements [7]. This is due to the differing activation functions of the hidden layer nodes. This article optimizes the initial parameter selection technique as well as the network training method based on this.

\subsubsection{Optimization of the $W N N$}

(1) Selection of Initial Values of Network Parameters. The initialization of network parameters has an impact on whether or not the network's subsequent learning converges and how quickly it converges. Currently, random values are used to create the initial parameters of WNNs, which significantly increases the number of learning times and even causes the network to fail to converge. The author suggested an autocorrelation correction initial parameter setting technique in [46], which links the initial parameter settings of WNN with wavelet types, wavelet time-frequency parameters, and learning samples. Excellent starting parameters may be obtained with a high degree of certainty using this approach, and the wavelet network's follow-up learning speed will be substantially increased. As a result, this article uses this approach to determine the initial values of network parameters, which will be discussed further in this article. 
(2) Determination of the Network Structure. In this section, we discuss the structure of our proposed Wavelet Neural Network based on optimized scaled conjugate gradient algorithm for the prediction of soft soil foundation engineering settlement.

(1) Number of hidden layers: the researcher of [47] demonstrated that a three-layer neural network model can handle general function fitting and approximation issues. Because settlement prediction is a function fitting issue, a three-layer wavelet neural network will suffice.

(2) Number of hidden layer nodes: the number of hidden layer nodes directly influences the network's generalization ability and training time; therefore, it is crucial in the development of the neural network model. There is, however, no theoretical direction in this area. The primary approaches used in practical applications are testing or the use of empirical equations [48].

This study presents an adaptive technique based on empirical formulas for obtaining a higher number of hidden layer nodes. To begin, use equation (1) to find the number of hidden layer nodes and the maximum number of learning periods for the network. When the network reaches its maximum number of nodes, the number of hidden layer nodes will increase since it is still unable to fulfill the error criteria. Similarly, when the network does not meet the learning number specified, and the error criterion is fulfilled concurrently, the number of hidden layer nodes will decrease.

$$
O=\sqrt{m+n}+1
$$

Here, $n$ is the number of input nodes, $m$ is the number of output nodes, is the number of hidden layer nodes, and $l$ is a constant between one and twenty.

3.2.3. Optimization of Learning Algorithm. Because the traditional BP network utilizes the steepest descent method, the primary drawback is that this slows down network convergence and is readily confined to the best local solution [41], too. For that reason, there were several optimization methods, among these; for its similarity in nature to the SDBP algorithm but with a higher convergence time, the connected gradient algorithm is frequently employed for tackling big optimization issues. For this reason, the SCG method [49] is used in this article for network training in the combined gradient algorithm. The following is the procedure of detailed application:

We take the error energy function as

$$
E(\theta)=\frac{1}{2} \sum(f(x, \theta)-y)^{2} .
$$

Here, $x$ is the input value, $y$ is the output value, and $\theta$ is a parameter.
For the objective function $E(\theta)$ of the wavelet neural network with $P$ input samples, the gradient of the $\theta$ (i.e. $\left.w_{k i}, w_{j k}, a_{k}, b_{k}\right)$ is

$$
\begin{aligned}
\frac{E}{w_{k i}} & =\sum_{p=1}^{P} \sum_{j=1}^{m}\left(f_{j}-y_{j}\right) w_{j k} \frac{\psi}{x \prime} x_{i}, \\
\frac{E}{w_{j k}} & =\sum_{p=1}^{P}\left(f_{j}-y_{j}\right) \psi\left(\frac{\sum_{i=1}^{n} w_{k i} x_{i}-b_{k}}{a_{k}}\right), \\
\frac{E}{a_{k}} & =\sum_{p=1}^{P} \sum_{j=1}^{m}\left(f_{j}-y_{j}\right) w_{j k} \frac{\psi}{a_{k}}, \\
\frac{E}{b_{k}} & =\sum_{p=1}^{P} \sum_{j=1}^{m}\left(f_{j}-y_{j}\right) w_{j k} \frac{\psi}{b_{k}} .
\end{aligned}
$$

If the Morlet wavelet function is used, namely, $\psi(t)=e^{\left(-t^{2} / 2\right)} \cos (5 t)$, then

$$
x^{\prime}=\sum_{i=1}^{n} w_{k i} x_{i}, t^{\prime}=\frac{x^{\prime}-b_{k}}{a_{k}} .
$$

Thus, the network parameters in equations (3) to (6) are

$$
\begin{aligned}
& \frac{\psi}{a_{k}}=\cos \left(5 t^{\prime}\right) e^{\left(-t^{t^{2}} / 2\right)} \frac{t^{t^{2}}}{a_{k}}+5 \sin \left(5 t^{\prime}\right) e^{\left(-t^{\prime^{2} / 2}\right)} \frac{t^{\prime}}{a_{k}}, \\
& \frac{\psi}{b_{k}}=\cos \left(5 t^{\prime}\right) e^{\left(-t^{t^{2}} / 2\right)} \frac{t^{\prime}}{a_{k}}+5 \sin \left(5 t^{\prime}\right) e^{\left(-t^{t^{2} / 2} / 2\right)} \frac{1}{a_{k}}, \\
& \frac{\psi}{t^{\prime}}=-\cos \left(5 t^{\prime}\right) e^{\left(-t^{t^{2}} / 2\right)} \frac{t^{\prime}}{a_{k}}-5 \sin \left(5 t^{\prime}\right) e^{\left(-t^{\prime^{2}} / 2\right)} \frac{1}{a_{k}} .
\end{aligned}
$$

Substituting these into the SCG algorithm, the optimal $\theta$ can be solved.

In the formulas above, $f_{j}(j=1,2, \ldots, m)$ is the output of the network, $\omega_{k i}$ is the connection weight between the $k$ th neuron in hidden layer and the $i$ th neuron in input layer, $\omega_{j k}$ is the connection weight between the $j$ th neuron in output layer and the $k$ th neuron in the hidden layer, and $a_{k}, b_{k}$ are the scale parameters and translation parameters of the wavelet basis function.

3.2.4. Optimized Construction of the WNN. Based on the foregoing research, the improved WNN model in this paper is constructed in the following steps:

Step 1: select an appropriate amount of training samples, define the training samples according to certain rules, and determine the number of input neurons and output neurons of the network.

Step 2: the learning algorithm of the network: SCG algorithm.

Step 3: set the network training period, target error, and other parameters. 
Step 4: calculate the number of hidden layer nodes in the network by empirical formulas, make adaptive optimization adjustments, and rebuild the network structure.

Step 5: transfer function: the hidden layer to the output layer adopts the sigmoid function, and the input layer to the hidden layer adopts the Morlet wavelet function.

Step 6: select a set of weights randomly and use the weights optimized by autocorrelation correction method as the initial weights for network training. Reset the training parameters of the network and use the SCG algorithm to train and establish an improved WNN.

3.2.5. Settlement Prediction Model. Equation (9) is used for sample normalization to make full use of the sensitivity of the Sigmoid function and improve the convergence speed of training [50].

$$
x^{*}=\frac{x-x_{\min }}{2\left(x_{\max }-x_{\min }\right)} .
$$

Here, $x_{\max }$ and $x_{\min }$ are the maximum and minimum values of each group of input components, and $x$ and $x^{*}$ are the values before and after normalization of each group of input components.

Apply the improved WNN described above.

Sample training: first, the measured samples $\left\{x_{i}, i=1,2, \ldots, n\right\}$ are divided into $k(k \in N, k \leq n)$ groups, and each group has $m+1(m=n-k)$ value. The first value is used as the input node value of the network, and the latter is used as the expected value of the output node. Next, the network connection weight is trained. Then, using the converged connection weight, $x_{k}, x_{k+1}, \ldots, x_{k+m-1}$ are used as the network input to calculate the predicted value $x_{k+m}$. At last, after removing $x_{k}$ and adding $x_{k+m}, x_{k+1}, x_{k+2}, \ldots, x_{k+m}$ are set as the new input of the network to calculate the predicted value $x_{k+m+1}$, and so on to make further predictions.

3.2.6. Training Plan. The three neural networks used have the same structure and training samples, and the differences are shown in Table 1. The training sample is the cumulative settlement value. The average of 10 times prediction will be the result to reduce the randomness of the predicted value. The model is evaluated by the relative error of the prediction result and the model accuracy. The model accuracy is given by

Model accuracy $=\sqrt{\frac{\sum \mid \text { predicted } \text { value }- \text { measured value }\left.\right|^{2}}{n-1}}$.

Here, $n$ is the number of predicted value.

Table 1 represents a comparison of three models such as BP Neural Network, which uses the SDBP Algorithm as a learning method, where Sigmoid is the function of hidden layer by randomly generating the initial parameter. While the SDBP Algorithm is used as the learning technique in the
BP Wavelet Neural Network, the Morlet Wavelet function of the hidden layer is generated randomly. Similarly, our Improved Wavelet Neural uses SCG Algorithm as a learning model instead of the SDBP Algorithm. The initial parameter in the case of our Improved Wavelet Neural Network is generating by the autocorrelation correction method.

\section{Experimental Work and Results}

Many soft foundation projects have emerged in recent years as a result of the steady building of national fundamental projects. Soft foundation engineering settlement prediction has always been a challenging topic in engineering due to the intricacy of soft foundation deformation. As a result, three neural networks are used to forecast the settlement of three common soft foundation projects: metro tunnels, roads, and high-rise structures, to compare and assess the convergence of the optimization model in this study.

4.1. Settlement Prediction of the Metro Tunnel. The west extension of one city's metro tunnel is located in a soft flowing murky salty clay layer with high moisture content, high compressibility, high sensitivity, low strength, and deformability. It is a floodplain of the Yangtze River with a thick covering layer, deep bedrock, and poor geological condition. As to the tunnel, its surrounding area is at the peak of the development period, there are many construction sites, and the settlement of its structure is obvious. The experimental data are 20 periods of the measurement points, which settled significantly. The first 15 periods of data are used as training samples to predict the settlement of the next 5 periods. The first 15 periods of data are divided into 8 training samples. Each group has 8 values: the first 7 values are used as the input of the network node, and the latter is used as the expected value of the output node. The prediction results of the three models are shown in Tables 2-5.

Table 2 illustrates the relative error and accuracy of settlement prediction of Metro Tunnel using BP Neural Network with training times 3503. During BP Neural Network, we obtain an accuracy of 2.45 for the number of measurements $16,17,18,19$, and 20 .

Table 3 explains the relative error and accuracy of settlement prediction of Metro Tunnel using WNN-based BP Algorithm with training times 931. During this algorithm, we obtain an accuracy of 1.32 for the number of measurements 16, 17, 18, 19, and 20 .

Table 4 describes the relative error and accuracy of settlement prediction of Metro Tunnel using Improved Wavelet Neural Network, with training times 267. During Improved Wavelet Neural Network, the accuracy of 0.89 for the number of measurements $16,17,18,19$, and 20 can be obtained.

Table 5 shows the relative error and accuracy of settlement prediction of Metro Tunnel using all the three techniques, with training times 3503, 931, and 267, respectively. This reflects that the model accuracy obtained during BP Neural Network, which is 2.45 , is greater than the WNN-based BP Algorithm and Improved WNN for the number of measurements 16,17, 18, 19, and 20 . 
Table 1: Comparison of three models.

\begin{tabular}{lcccc}
\hline S. no. & Model & Learning method & Hidden layer function & Initial parameter \\
\hline 1 & BP neural network & SDBP algorithm & Sigmoid function & Randomly generated \\
2 & BP wavelet neural network & SDBP algorithm & Morlet wavelet function & Randomly generated \\
3 & Improved wavelet neural network & SCG algorithm & Morlet wavelet function & Autocorrelation correction method \\
\hline
\end{tabular}

TABLE 2: Settlement prediction of the metro tunnel using the BP neural network.

\begin{tabular}{lccc}
\hline S. no. & Measurement number & Training times & Relative error (\%) \\
\hline 1 & 16 & & 0.152 \\
2 & 17 & 3503 & 0.343 \\
3 & 18 & & 0.164 \\
4 & 19 & 0.109 & 0.278 \\
5 & 20 & 2.45 \\
\hline
\end{tabular}

TABLE 3: Settlement prediction of the metro tunnel using WNN-based BP algorithm.

\begin{tabular}{lccc}
\hline S. no. & Measurement number & Training times & Relative error (\%) \\
\hline 1 & 16 & & 0.079 \\
2 & 17 & 931 & 0.073 \\
3 & 18 & & 0.118 \\
4 & 19 & 0.060 \\
5 & 20 & 0.200 \\
\hline
\end{tabular}

Table 4: Settlement prediction of the metro tunnel using the improved wavelet neural network.

\begin{tabular}{lccc}
\hline S. no. & Measurement number & Training times & Relative error (\%) \\
\hline 1 & 16 & & 0.058 \\
2 & 17 & 267 & 0.095 \\
3 & 18 & 0.103 \\
4 & 19 & 0.029 \\
5 & 20 & 0.095 \\
\hline
\end{tabular}

TABLE 5: Settlement prediction of the metro tunnel using all three techniques.

\begin{tabular}{|c|c|c|c|c|c|c|}
\hline \multirow{2}{*}{ Measurement number } & \multicolumn{2}{|c|}{ BP neural network } & \multicolumn{2}{|c|}{ WNN-based BP algorithm } & \multicolumn{2}{|c|}{ Improved wavelet neural network } \\
\hline & Training times & Relative error (\%) & Training times & Relative error (\%) & Training times & Relative error (\%) \\
\hline 16 & & 0.152 & & 0.079 & & 0.058 \\
\hline 17 & & 0.343 & & 0.073 & & 0.095 \\
\hline 18 & 3503 & 0.164 & 931 & 0.118 & 267 & 0.103 \\
\hline 19 & & 0.109 & & 0.060 & & 0.029 \\
\hline 20 & & 0.278 & & 0.200 & & 0.095 \\
\hline Model accuracy & & 2.45 & & 1.32 & & 0.89 \\
\hline
\end{tabular}

4.2. Settlement Prediction of the Highway Soft Soil Roadbed. Yangtze River Bridge, opened to traffic in 2001, is one of the national key construction projects during the ninth five-year plan period. Its lead is a soft soil foundation, and settlement monitoring points are laid out according to its sections. The 13 periods of monitoring data from a monitoring point on a certain section are selected for prediction experiment, among which the first 9 period's data are used as training samples to predict the settlement of the last 4 periods. The settlement date of the first 9 periods is divided into 4 training samples. Each group has 6 values: the first 5 values are used as the input of the network node, and the latter is used as the expected value of the output node. The three network models are used to predict respectively; the results are given in Tables 6-9.

Table 6 illustrates the relative error and accuracy of settlement prediction of soft soil roadbed of highway using BP Neural Network with training times 2589. During BP Neural Network, we obtain an accuracy of 6.10 for the number of measurements $10,11,12$, and 13 . 
TABLE 6: Settlement prediction of the soft soil roadbed of the highway using the BP neural network.

\begin{tabular}{lccc}
\hline S. no. & Measurement number & Training times & Relative error (\%) \\
\hline 1 & 10 & & 0.216 \\
2 & 11 & 2589 & 0.123 \\
3 & 12 & & 0.206 \\
4 & 13 & & 0.172 \\
& & & 6.10 \\
\hline
\end{tabular}

TABLE 7: Settlement prediction of the soft soil roadbed of the highway using WNN-based BP algorithm.

\begin{tabular}{lccc}
\hline S. no. & Measurement number & Training times & Relative error (\%) \\
\hline 1 & 10 & & 0.128 \\
2 & 11 & 616 & 0.096 \\
3 & 12 & & 0.112 \\
4 & 13 & & 0.103 \\
\hline
\end{tabular}

TABLE 8: Settlement prediction of the soft soil roadbed of the highway using the improved wavelet neural network.

\begin{tabular}{lccc}
\hline S. no. & Measurement number & Training times & Relative error (\%) \\
\hline 1 & 10 & & 0.015 \\
2 & 11 & 132 & 0.096 \\
3 & 12 & & 0.055 \\
4 & 13 & & 0.054 \\
& & & 1.82 \\
\hline
\end{tabular}

Table 7 describes the relative error and accuracy of settlement prediction of soft soil roadbed of highway using WNN-based BP Algorithm with training times 616. During this technique, we obtain an accuracy of 3.65 for the number of measurements $10,11,12$, and 13 .

Table 8 explains the relative error and accuracy of settlement prediction of soft soil roadbed of highway using Improved Wavelet Neural Network, with training times 132. During Improved Wavelet Neural Network, the accuracy of 1.82 for the number of measurements $10,11,12$, and 13 can be obtained.

Table 9 shows the relative error and accuracy of settlement prediction of soft soil roadbed of highway using all the three techniques, with training times 2589,616 , and 132 , respectively. This reflects that the model accuracy obtained during BP Neural Network is 6.10 for the number of measurements $10,11,12$, and 13 . This reflects that this accuracy is greater than the WNN-based BP Algorithm and Improved WNN.

4.3. Settlement Prediction of the Building Foundation. The 21 periods monitoring data of a high-rise building soft foundation are taken for analysis. The first 13 periods of data are used as training samples to predict the settlement of the last 8 periods of observation. Use the three network models to make predictions, and the results are listed in Tables 10-13.

Table 10 illustrates the relative error and accuracy of settlement prediction of building foundation using BP Neural Network with training times 3201. During BP Neural Network, we obtain accuracy of 0.43 for the number of measurements $14,15,16,17,18,19,21$, and 21 .
Table 11 describes the relative error and accuracy of settlement prediction of building foundation using WNNbased BP Algorithm with training times 1145. During this technique, we obtain an accuracy of 0.47 for the number of measurements $14,15,16,17,18,19,20$, and 21 .

Table 12 shows the relative error and accuracy of settlement prediction of building foundation using Improved Wavelet Neural Network, with training times 254. During Improved Wavelet Neural Network, the accuracy of 0.35 for the number of measurements $14,15,16,17,18,19,20$, and 21 can be obtained.

Table 13 shows the relative error and accuracy of settlement prediction of building foundation using all the three techniques, with training times 3201, 1145, and 254, respectively. This reflects that the model accuracy obtained during WNN-based BP Algorithm is 0.47 for the number of measurements $14,15,16,17,18,19,20$, and 21 . This reflects that this accuracy is greater than the rest of the two techniques.

Figures 3-5 show a comparison of the three techniques, where the mean relative error and maximum absolute error of the prediction results obtained using the BP neural network model are larger than those obtained using the WNN-based BP Algorithm. It demonstrates that the WNNbased BP Algorithm's generalization (prediction) capacity outperforms the BP neural network model. The forecast findings from the Improved WNN approach are larger than the measured settlement values, which is consistent with the actual engineering experience. When these two approaches are compared, the prediction power of the WNN-based BP Algorithm is superior to that of the Improved WNN method. 
TABLE 9: Settlement prediction of the soft soil roadbed of the highway using all three techniques.

\begin{tabular}{lccccc}
\hline \multirow{2}{*}{ Measurement number } & \multicolumn{2}{c}{ BP neural network } & \multicolumn{2}{c}{ WNN based on BP algorithm } & \multicolumn{2}{c}{$\begin{array}{c}\text { Improved wavelet neural network } \\
\text { Training times }\end{array}$} & Relative error (\%) & Training times & Relative error (\%) & Training times & Relative error (\%) \\
\hline 10 & & 0.216 & & 0.128 & 0.015 \\
11 & 2589 & 0.123 & 616 & 0.096 & 0.096 \\
12 & & 0.206 & & 0.112 & 0.055 \\
13 & & 0.172 & & 3.65 & 0.054 \\
Model accuracy & 6.10 & & & 1.82 \\
\hline
\end{tabular}

TABLE 10: Settlement prediction of the building foundation using the BP neural network.

\begin{tabular}{lccc}
\hline S. no. & Measurement number & Training times & Relative error (\%) \\
\hline 1 & 14 & 0.096 \\
2 & 15 & 0.018 \\
3 & 16 & 3201 & 0.048 \\
4 & 17 & 0.011 \\
5 & 18 & 0.012 \\
6 & 19 & 0.014 \\
7 & 20 & 0.061 \\
8 & 21 & 0.035 \\
\hline
\end{tabular}

TABLE 11: Settlement prediction of the building foundation using WNN-based BP algorithm.

\begin{tabular}{lrrr}
\hline S. no. & Measurement number & Training times & Relative error (\%) \\
\hline 1 & 14 & & 0.060 \\
2 & 15 & & 0.056 \\
3 & 16 & 1145 & 0.070 \\
4 & 17 & & 0.037 \\
5 & 18 & 0.009 \\
6 & 19 & 0.001 \\
7 & 20 & 0.031 \\
8 & 21 & 0.088 \\
\end{tabular}

TABLE 12: Settlement prediction of the building foundation using the improved wavelet neural network.

\begin{tabular}{|c|c|c|c|}
\hline S. no. & Measurement number & Training times & Relative error (\%) \\
\hline 1 & 14 & \multirow{8}{*}{254} & 0.025 \\
\hline 2 & 15 & & 0.043 \\
\hline 3 & 16 & & 0.053 \\
\hline 4 & 17 & & 0.024 \\
\hline 5 & 18 & & 0.029 \\
\hline 6 & 19 & & 0.014 \\
\hline 7 & 20 & & 0.010 \\
\hline 8 & 21 & & 0.071 \\
\hline \multicolumn{2}{|c|}{ Model accuracy } & & \\
\hline
\end{tabular}

The WNN outperforms the BP neural network in terms of prediction accuracy and convergence speed and has excellent adaptive prediction capabilities when compared to the WNN based on the SDBP algorithm, according to settlement prediction findings for three types of soft foundation engineering. As a result, the improved WNN based on the SCG algorithm greatly increases prediction accuracy and convergence speed. 
TABLE 13: Settlement prediction of the building foundation using all three techniques.

\begin{tabular}{lccccc}
\hline \multirow{2}{*}{ Measurement number } & \multicolumn{2}{c}{ BP neural network } & \multicolumn{2}{c}{ WNN-based BP algorithm } & \multicolumn{2}{c}{$\begin{array}{c}\text { Improved wavelet neural network } \\
\text { Training times }\end{array}$} & Relative error (\%) & Training times & Relative error (\%) & Training times & Relative error (\%) \\
\hline 14 & & 0.096 & & 0.060 & 0.025 \\
15 & & 0.018 & & 0.056 & 0.043 \\
16 & \multirow{2}{*}{3201} & 0.048 & & 0.070 & 0.053 \\
17 & & 0.011 & 1145 & 0.037 & 0.024 \\
18 & & 0.012 & & 0.009 & 0.029 \\
19 & & 0.014 & & 0.001 & 0.014 \\
20 & & 0.061 & 0.031 & 0.010 \\
21 & & 0.035 & & 0.47 & 0.071 \\
Model accuracy & 0.43 & & & \\
\hline
\end{tabular}

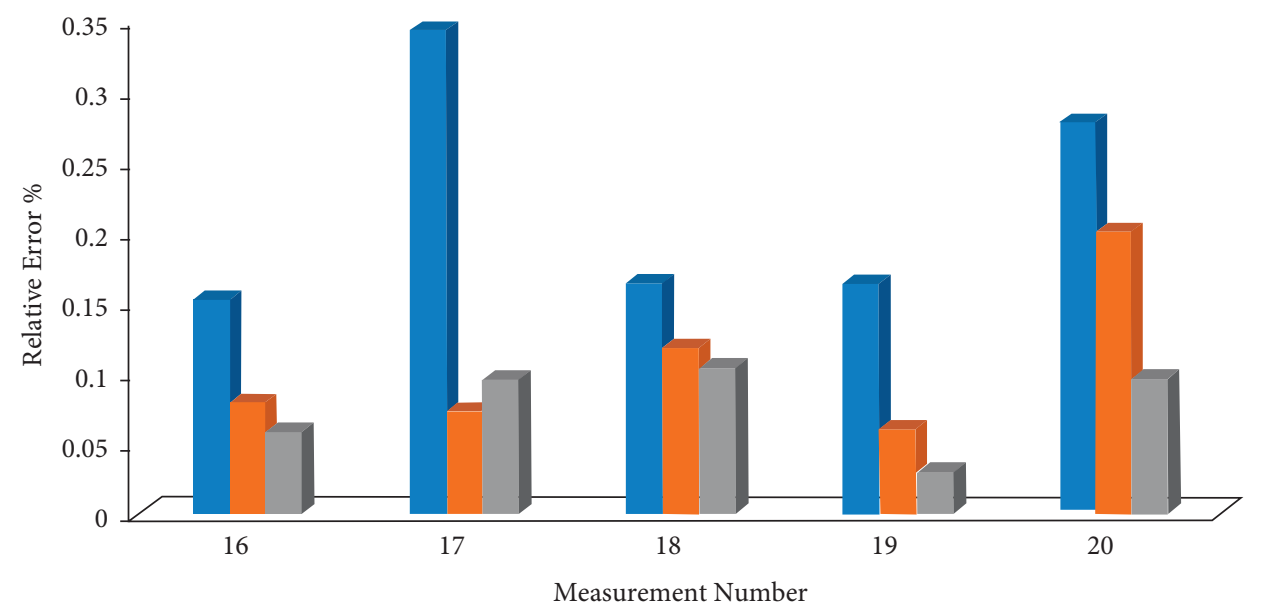

- BP Neural Network

- WNN based BP Algorithm

Improved WNN

Figure 3: Settlement prediction of the metro tunnel.

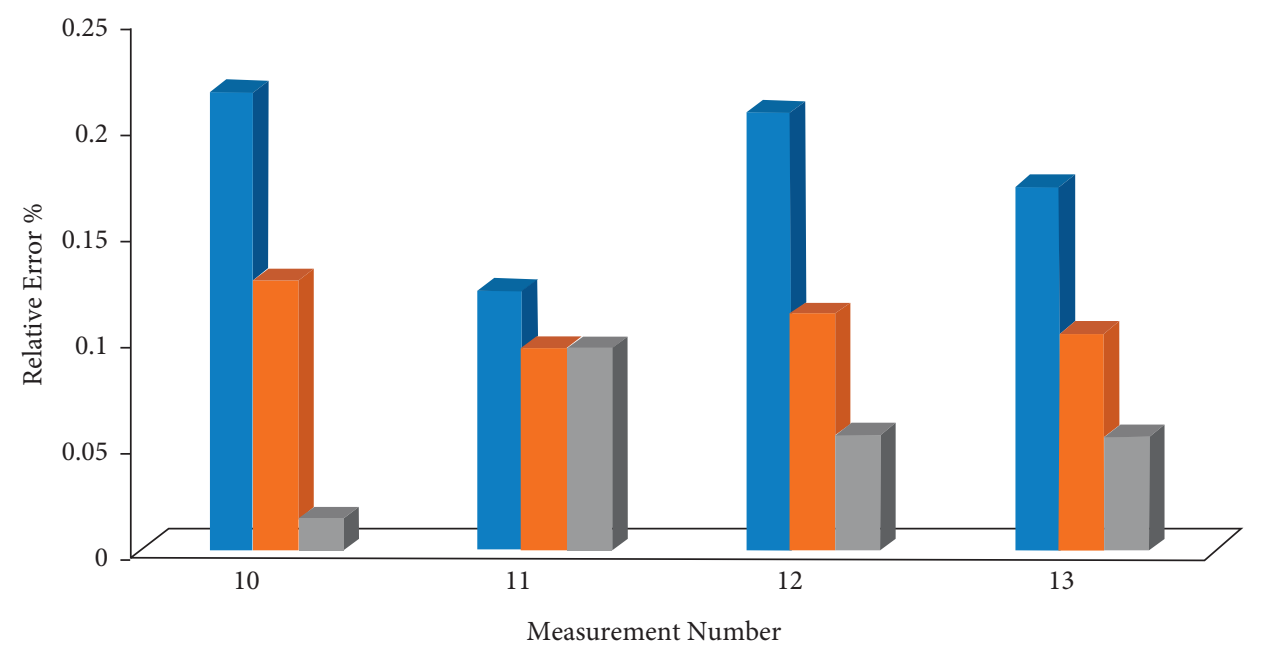

n BP Neural Network

- WNN based BP Algorithm

- Improved WNN

FIGURE 4: Settlement prediction of the soft soil roadbed of the highway. 


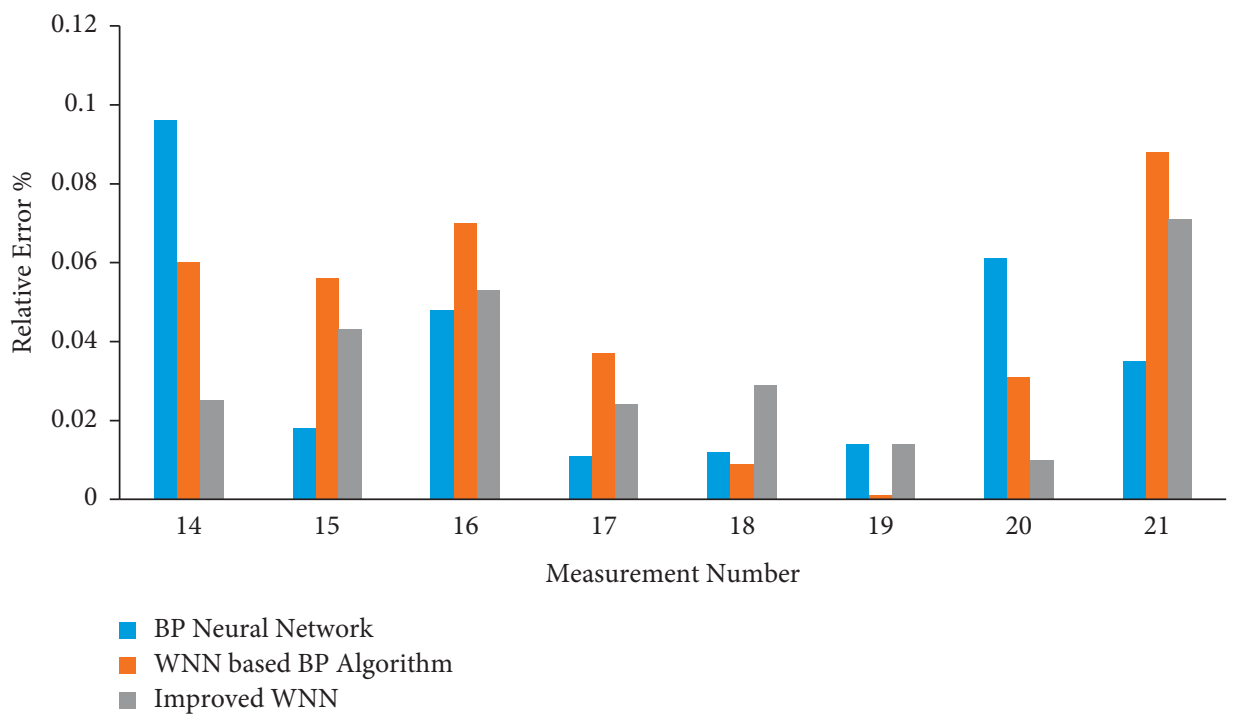

FIGURE 5: Settlement prediction of the building foundation.

\section{Conclusion}

Aiming at the convergence defects in the application of traditional settlement prediction models, this paper optimizes the WNN based on the traditional BP algorithm and applies it to soft foundation engineering. Here, we have introduced BP Neural Network and Improved Wavelet Neural Network in the soft ground foundation engineering forecast based on the basic concept of the wavelet neural network. The neural wavelet network method has been enhanced, and the scale conjugate gradient technique has been updated to maximize the excellent value neural wavelet network approach. The enhanced wavelet neural network settlement model is constructed, and settlements are predicted based on the conventional BP technique. The improved wavelet neural network model based on the classic BP method has greater prediction accuracy, and the soft soil settling has a good prediction effect with a specified reference value, according to the comparison of calculation accuracy evaluation indexes.

\section{Data Availability}

The datasets used and/or analyzed during the current study are available from the corresponding author upon reasonable request.

\section{Conflicts of Interest}

The authors declare that they have no conflicts of interest.

\section{Authors' Contributions}

Guihua Li and Chenyu Han designed the study, analyzed the data, and wrote the manuscript. Hong Mei and Shuai Chen analyzed the data and contributed to writing the manuscript.

\section{Acknowledgments}

This study was supported by the Fundamental Research Funds for the Central Universities (B210205013).

\section{References}

[1] P. Tao, Y. An-ying, L. Xing, and Y. Qin, "Prediction of soft ground settlement based on BP neural network-grey system united model," Rock and Soil Mechanics, vol. 26, no. 11, pp. 1810-1814, 2005.

[2] T.-sheng Wang, Li-ping Zhang, and Xi-sheng Hua, "Review on research of deformation prediction of metro tunnel construction," Advances in Science and Technology of Water Resources, vol. 5, pp. 62-65, 2003.

[3] H. Shen-xiang, H. Yin, and Z. Jiang, Deformation Monitoring Data Processing and Methods, Wuhan University Press, Wuhan, China, 2003.

[4] Z. Guo-qiang, H. Wang, Li Li, W. Cheng-tang, and X. I. E. Biting, "Prediction of maximum settlement of foundation pit based on SFLA-GRNN model," Rock and Soil Mechanics, vol. 40, no. 2, pp. 792-798, 2019.

[5] X. Xia, X. Liu, and J. Lou, "A network traffic prediction model of smart substation based on IGSA-WNN," ETRI Journal, vol. 42, no. 3, pp. 366-375, 2020.

[6] Na Yang, Q. Fu, W. Shu-li, and Li Rong-dong, "Improvement of wavelet neural networks model and application," Journal of Systems Science \& Information, vol. 29, no. 1, pp. 168-173, 2009.

[7] Bi Yan-qiu, W.-lian Zhou, and Da-hai Zhang, "Investigation on the convergence performance of continuous wavelet neural network," Proceedings of the CSU-EPSA, vol. 12, no. 6, pp. 51-53, 2005.

[8] Y. Zhang, H. Yang, H. Cui, and Q. Chen, "Comparison of the ability of ARIMA, WNN and SVM models for drought forecasting in the Sanjiang Plain, China," Natural Resources Research, vol. 29, no. 2, pp. 1447-1464, 2020.

[9] Q. Qinghua Zhang, "Using wavelet network in nonparametric estimation," IEEE Transactions on Neural Networks, vol. 8, no. 2, pp. 227-236, 1997. 
[10] J. Qian, R. Zhou, S. Chen, X. Gu, and M. Huang, "Influence of pavement roughness on dynamic stresses in saturated subsoil subjected to moving traffic loading," International Journal of Geomechanics, vol. 18, no. 4, Article ID 04018012, 2018 b.

[11] X. Yu, F. Jiang, J. Du, and D. Gong, "A cross-domain collaborative filtering algorithm with expanding user and item features via the latent factor space of auxiliary domains," Pattern Recognition, vol. 94, pp. 96-109, 2019.

[12] J. Yu and R. Yang, "Study on the predictive algorithm of plant restoration under heavy metals," Scientific Programming, vol. 2021, Article ID 6193182, 2021.

[13] R. A. Barron, "Closure of "consolidation of fine-grained soils by drain wells"," Transactions of the American Society of Civil Engineers, vol. 113, no. 6, pp. 751-754, 1948.

[14] L. Kok, M. Jamiolkowski, and S. Hansbo, "Consolidation by vertical drains," G'eotechnique, vol. 31, no. 31, pp. 45-66, 1981.

[15] Q. Zheng, A. C. F. Chiu, G. H. Lei, and C. W. W. Ng, “An analytical solution for consolidation with vertical drains under multiramp loading," G'eotechnique, vol. 65, no. 7, pp. 531-547, 2015.

[16] T. Yang, G. W. Li, and W. Q. Yang, "Settlement prediction of stage constructed embankment on soft ground based on the hyperbolic method," Rock and Soil Mechanics, vol. 25, no. 10, pp. 1551-1554, 2004, in Chinese.

[17] S. Y. Liu and F. Jing, "Settlement prediction of embankments with stage construction on soft ground," Chinese Journal of Geotechnical Engineering, vol. 25, no. 2, pp. 228-232, 2003.

[18] M. V. Ribeiro, C. A. Duque, and J. M. T. Romano, "An interconnected type-1 fuzzy algorithm for impulsive noise cancellation in multicarrier-based power line communication systems," IEEE Journal on Selected Areas in Communications, vol. 24, no. 7, pp. 1364-1376, 2006.

[19] J.-S. R. Jang, "ANFIS: adaptive-network-based fuzzy inference system," IEEE Transactions on Systems, Man, and Cybernetics, vol. 23, no. 3, pp. 665-685, 1993.

[20] I. Daubechies, Ten Lectures on Wavelets, Vol. 61, Society for Industrial and Applied Mathematics, Philadelphia, PA, USA, 1992.

[21] M. Yu, T. Quan, Q. Peng, X. Yu, and L. Liu, "A model-based collaborate filtering algorithm based on stacked autoencoder," Neural Computing \& Applications, vol. 6, no. 12, 2021.

[22] Y. C. Pati and P. S. Krishnaprasad, "Analysis and synthesis of feedforward neural networks using discrete affine wavelet transformations," IEEE Transactions on Neural Networks, vol. 4, no. 1, pp. 73-85, 1993.

[23] Q. Zhang and A. Benveniste, "Wavelet networks," IEEE Transactions on Neural Networks, vol. 3, no. 6, pp. 889-898, 1992.

[24] C. Bernard, S. Mallat, and J. jacques Slotine, "Wavelet interpola- tion networks," in Preprint, Centre de Mathematiques AppliqueesEcole Polytechnique, 1999.

[25] A. K. Alexandridis and A. D. Zapranis, "Wavelet neural networks: a practical guide," Neural Networks, vol. 42, pp. 1-27, 2013.

[26] F. Shin, K. Takayama, and T. Hachisuka, "Wavelet convolutional neural networks for texture classification," arXiv preprint arXiv:1707.07394, 2017.

[27] H. Huang, R. He, Z. Sun, and T. Tan, "Wavelet-srnet: a wavelet- based cnn for multi-scale face super resolution," in Proceedings of the IEEE Conference on Computer Vision and Pattern Recognition, pp. 1689-1697, Silver Spring, MD, USA, July 2017.
[28] T. Guo, H. S. Mousavi, T. H. Vu, and V. Monga, "Deep wavelet prediction for image superresolution," in Proceedings of the IEEE Conference on Computer Vision and Pattern Recognition (CVPR) Workshops, Seattle, WA, USA, July 2017.

[29] S. F. Ddn De Silva, I. T. S. Piyatilake, and A. V. S. Karunarathne, "Wavelet based edge feature enhancement for convolutional neural networks," International Conference in Machine Vision, vol. 11041, 2018.

[30] P. Liu, H. Zhang, K. Zhang, L. Lin, and W. Zuo, "Multi-level wavelet-cnn for image restoration," 2018. arXiv preprint arXiv:1805.07071.

[31] B. Zhang and L. Meng, "Energy efficiency analysis of wireless sensor networks in precision agriculture economy," Scientific Programming, vol. 2021, Article ID 8346708, 2021.

[32] E. Oyallon, E. Belilovsky, and S. Zagoruyko, "Scaling the scattering transform: deep hybrid networks," in Proceedings of the International Conference on Computer Vision (ICCV), Venice, Italy, October 2017.

[33] S. Mallat, "Group invariant scattering," Communications on Pure and Applied Mathematics, vol. 65, no. 10, pp. 1331-1398, 2012.

[34] X. Yu, Q. Peng, L. Xu, F. Jiang, J. Du, and D. Gong, “A selective ensemble learning based two-sided cross-domain collaborative filtering algorithm," Information Processing \& Management, vol. 58, no. 6, Article ID 102691, 2021.

[35] B. Alizadeh Savareh, E. Hassan, M. Hajiabadi, S. Majid Azimi, and M. Ghafoori, "Wavelet-enhanced convolutional neural network: a new idea in a deep learning paradigm," Biomedical Engineering/Biomedizinische Technik, vol. 64, no. 2, pp. 195205, 2018.

[36] L. Rui, X. Xiaoyu, and D. Xueyan, "Fatigue load spectrum of highway bridge vehicles in plateau mountainous area based on wireless sensing," Mobile Information Systems, vol. 2021, Article ID 9955988, 2021.

[37] L. Ma, J. "org Stu"ckler, T. Wu, and D. Cremers, "Detailed dense inference with convolutional neural networks via discrete wavelet transform," arXiv preprint arXiv:1808.01834, 2018.

[38] Z. Zhang, Y. Shi, H. Toda, and T. Akiduki, "A study of a new wavelet neural network for deep learning," in Proceedings of the Wavelet Analysis and Pattern Recognition (ICWAPR), 2017 International Conference on, pp. 127-131, IEEE, Xian, China, July 2017.

[39] S. Bo and B. Kexin, "Open innovation mode of green innovation system for manufacturing industry," Mobile Information Systems, vol. 2021, Article ID 9948683, 2021.

[40] G. Li, T. Huang, M. Jiang, and R. Yue, "Optimization and application research of wavelet neural network," in Proceedings of the 2009 International Workshop on Intelligent Systems and Applications, Wuhan, China, May 2009.

[41] L. Guo-dong and D. Jin, "Discussion on problems of BP neural networks applied to hydrological prediction," Journal of Hydraulic Engineering, vol. 1, pp. 65-70, 1999.

[42] X. Ni, "Discussion on soft soil foundation treatment technology in municipal road and bridge engineering," E3S Web of Conferences, vol. 165, Article ID 04002, 2020.

[43] J. Lin, "Discussion on the technical characteristics of soft ground foundation treatment in municipal road and bridge engineering construction," Henan Building Materials, vol. 3, pp. 224-225, 2019.

[44] F. Lu, J. H. Xu, and Z. Y. Wang, "Study on genetic wavelet neural network model of water demand prediction in Hefei City," Science of Surveying and Mapping. Papers, vol. 38, no. 5, pp. 28-31, 2013. 
[45] J. Y. Hu, H. Y. Wen, and L. Zhou, "Application of genetic wavelet neural network in prediction of dam deformation," Yellow River. Papers, vol. 36, no. 10, pp. 126-128, 2014.

[46] Z. Xue-zhi, Z. Chun-hua, and C. Tong-jian, "A research on the initialization of parameters of wavelet neural networks," Journal of South China University of Technology, vol. 2, no. 2, pp. 77-79, 2003.

[47] K.-l. Hsu, H. V. Gupta, and S. Sorooshian, "Artificial neural network modeling of the rainfall-runoff process," Water Resources Research, vol. 31, no. 10, pp. 2517-2530, 1995.

[48] B. Ye and L. Yan, "Analysis of choosing the number of the hiddenlayers and its nodes number in back propagation network," Journal of Shangqiu Vocational and Technical College, vol. 12, no. 6, pp. 52-53, 2004.

[49] S. O. Sada, "Improving the predictive accuracy of artificial neural network (ANN) approach in a mild steel turning operation," International Journal of Advanced Manufacturing Technology, vol. 112, no. 9-10, pp. 2389-2398, 2021.

[50] W. Bi and G. Wang, "Local cultural IP development and cultural creative design based on big data and internet of things," Mobile Information Systems, vol. 2021, Article ID 5521144, 2021. 